\title{
Improved SAR Image Coregistration Using Pixel-offset Series
}

\author{
Teng Wang, Sigurjón Jónsson and Ramon F. Hanssen
}

\begin{abstract}
Synthetic Aperture Radar (SAR) image coregistration is a key procedure before Interferometric SAR (InSAR) time-series analysis can be started. However, many geophysical datasets suffer from severe decorrelation problems due to a variety of reasons, making precise coregistration a non-trivial task. Here, we present a new strategy that uses pixeloffset series of detected sub-image patches dominated by point-like targets to improve SAR image coregistrations. First, all potentially coherent image pairs are coregistered in a conventional way. In this step, we propose a coregistration quality index for each image to rank its relative "significance" within the dataset and to select a reference image for the SAR dataset. Then, pixel-offset series of detected point-like targets are made from amplitude maps to improve the geometrical mapping functions. Finally, all images are resampled depending on the pixel-offsets calculated from the updated geometrical mapping functions. We used images from a rural region near the North Anatolian Fault in eastern Turkey to test the proposed method and clear coregistration improvements were found based on amplitude stability. This enhanced coregistration strategy should therefore lead to improved InSAR time-series analysis results.
\end{abstract}

\section{Index Terms}

SAR, Image coregistration, Pixel offsets, InSAR.

\section{INTRODUCTION}

Interferometric Synthetic Aperture Radar (InSAR) time-series analysis techniques are widely used in earth sciences to measure deformation patterns and trends of the Earth's crust at high spatial resolution [1]-[4]. In time-series InSAR processing, SAR images are usually coregistered and resampled onto the same grid of a chosen dataset reference image (master) before forming interferograms. Imprecise coregistration reduces interferometric coherence and can consequently cause poor time-series results [5]-[7]. While Small-Baseline (SB) coregistration strategies, such as in [2], [8], are often used to facilitate the coregistration of all the images in a dataset, difficulties in coregistering severely decorrelated image pairs in many datasets remain. Generally speaking, there are three situations in which such decorrelated image pairs become problematic: 1) coregistration between long temporal

T. Wang and S. Jónsson are with King Abdullah University of Science and Technology, Thuwal, Saudi Arabia. (e-mail: teng.wang@kaust.edu.sa)

R. F. Hanssen is with Delft University of Technology, Delft, the Netherlands. (e-mail: R.F.Hanssen@tudelft.nl)

Manuscript received July, 2013. 
and/or normal baseline pairs, 2) coregistration between SAR images spanning a strong deformation event, such as a volcanic eruption or a large earthquake, and 3) coregistration between SAR images with seasonal feature changes, for example, with and without snow coverage. Current research efforts on SAR image coregistration between poorly correlated image pairs can be divided into two main branches. The first branch of methods seeks to detect features (usually point-like targets) in the SAR images to estimate pixel-offsets [9]-[12], while the second branch focuses on developing geometrical mapping functions with which pixel-offsets are calculated pixel-by-pixel using the given orbital information and a Digital Elevation Model (DEM) [13], [14].

In the first branch of methods, point-like targets (PTs) are typically detected by searching for local maxima from the 2-D cross-correlation surface calculated between oversampled SAR images and an ideal 2-D impulse response template (usually a sinc-like function). Then, the pixel offsets are estimated from the location differences of the detected PTs [11]. Although high precision offset measures can be obtained on such PTs, the problem is that the topography-related range-offsets are not adequately modeled by a low-order polynomial mapping function [14]. Even with a given DEM, the topography-related range-offsets are also related to the changes in looking angle and satellite position during image acquisitions. Within the second branch of methods, topography is accounted for in the geometrical mapping function, although some tie points are still needed to reduce the systematic errors in that mapping function. In summary, both reliable pixel-offset estimates and appropriate mapping functions are highly beneficial, and it is therefore important to combine the two branches of methods to better coregister time-series SAR images. The combination can be carried out by using pixel offsets estimated from isolated PTs to correct the geometrical mapping functions in urban sites [11]. However, in geophysical studies, processing of multi-frame images extending 100s of km across rural plate boundary zones are often needed. For such cases, it can be difficult to obtain enough reliable pixel-offset estimates between image pairs with large normal and temporal baseline separations, given the fact that the systematic errors of the geometrical mapping function can hardly be treated as a constant shifts [15].

Aiming at overcoming these difficulties, we propose a two-step strategy that first estimates pixel-offset series from detected PT sub-images (match-patches, or chips) and then uses them to refine geometrical mapping functions. In particular, we first estimate pixel-offsets between all potentially coherent image pairs using the standard approach, i.e. by cross-correlating a certain number of uniformly distributed match-patches and then resample all the slave images using polynomial mapping functions estimated from the pixel-offset estimates [2], [16]. In this step, a coregistration quality index is introduced to assess the relative significance of the images in the dataset. We then use the coregistered images to detect match-patches dominated by a PT, on which the pixel-offsets are re-estimated. For each PT, we generate a pixel-offset series that is related to the same physical target on the ground. Finally, we resample all the original slave images onto the reference grid using geometrical mapping functions that have been corrected by the pixel-offset series. We tested our method by using 29 two-frame ENVISAT Advanced SAR (ASAR) images of a rural site across the North Anatolian Fault (NAF) in eastern Turkey, where both steep topography and seasonal snow coverage complicate the SAR image coregistration. 


\section{INITIAL COREGISTRATION AND QUALITY INDEX}

In our image coregistration strategy, the starting point is similar to the SB-coregistration method that is used in [2]. We estimate pixel-offsets between potentially coherent image-pairs on a number of match-patches that are uniformly distributed throughout the two scenes. We then estimate parameters $\vec{\alpha}^{s, m}$ of a 2-D polynomial mapping function to model the pixel-offset field between a slave image $s$ and a master image $m$ :

$$
\vec{\alpha}^{s, m}=\left(P^{T} W P\right)^{-1} P^{T} W \boldsymbol{\Delta}_{\mathrm{amp}}^{s, m}\left(i_{k}, j_{k}\right)
$$

where $\boldsymbol{\Delta}_{\text {amp }}^{s, m}\left(i_{k}, j_{k}\right)$ is the pixel offset estimated on match-patch $k$, located at azimuth and range coordinates $i$ and $j$. For a quadric polynomial mapping function, each row of matrix $P$ is: $\left[\begin{array}{cccccc}1 & i & j & i^{2} & i j & j^{2}\end{array}\right]$, while $W$ is a diagonal weight matrix whose elements are the cross-correlation magnitude peak values $c_{k}$ from the pixel-offset estimations.

The procedures described above can be regarded as the first step in obtaining overall coherence information between SAR images. As there is a close relation between amplitude cross-correlation and complex coherence [7], the number of successfully coregistered match-patches can be used as an indication of the overall coherence between image pairs [8]. However, the spatial distribution of the match-patches is also important and should be considered as well in assessing the quality of the coregistration. For this task, we adopt the concept of Dilution of Precision (DOP) from GPS, which can be obtained from the coefficient inversion in (1) [18]:

$$
\mathrm{DOP}=\sum\left|\operatorname{diag}\left(\left(P^{T} W P\right)^{-1}\right)\right|
$$

where $P$ and $W$ are the same matrices as above. The name dilution of precision comes from the fact that the measurement errors in $\boldsymbol{\Delta}_{\text {amp }}^{s, m}\left(i_{k}, j_{k}\right)$ are propagated into the least-squares solution $\vec{\alpha}$ through $\left(P^{T} W P\right)^{-1}$. As $P$ is only a function of the match-patch location, given the same number of match-patches, their spatial distribution generally leads to different DOP values. For instance, if all the match-patches are located in a small area (i.e. a large DOP value [18]), then the mapping function coefficient estimation will likely be unreliable. Finally, using the cross-correlation results between images and the DOP, we define a quantitative measure to evaluate the quality of the coregistration, a Coregistration Quality Index (CQI), which we define as:

$$
\mathrm{CQI}=\frac{\sum_{k=1}^{L} c_{k}}{\mathrm{DOP}}
$$

where $c_{k}$ is the amplitude cross-correlation value on the $k^{t h}$ match-patch. The numerator of (3) includes both the number and quality (the magnitude of $c_{k}$ ) of the match-patches while the denominator indicates the spatial distribution of them. Note that the CQI can be large when DOP is very small, and therefore we normalize the CQIs using the maximum CQI and obtain the relative CQIs. We also define an empirical CQI threshold to identify coregistration outliers and remove such outlier pairs from the coregistration network ( 0.1 in our case study), while keeping the quality image-pairs for the mapping function inversion.

Moreover, the coregistration graph can be represented as an $M \times N$ 'incidence matrix' $A$, a sparse matrix with $-1 \mathrm{~s}$ and $1 \mathrm{~s}$ indicating master and slave images. By putting the CQI value of each image pair on the diagonal of 
a matrix, $Q$, we can obtain a quality index vector, $\vec{q}$, whose elements represent the average CQIs associated with each image: $\vec{q}=\frac{\operatorname{diag}\left(A^{T} Q A\right)}{\operatorname{diag}\left(A^{T} A\right)}$. The derived image quality index vector $\vec{q}$ for the dataset images is a useful indicator for evaluating the "significance" of each image (Fig. 1). It is interesting to note that all images with very low $q$ (dark blue squares in Fig. 1) were acquired during winter months and their low quality index can thus be explained by the snow coverage in this mountainous region. The image with the largest $q$ value is a natural choice as the dataset reference image rather than selecting it from a coherence model prediction [2]. For example, the coherence model predicts that image \#11 of the NAF dataset would be the best choice as the dataset reference image, but this image was acquired in winter and has a very low $q$ value. If we would blindly select this image as the dataset reference image, all the subsequent analysis would be affected. Although we could manually avoid using winter images to improve the time-series analysis results, the quality index quantitatively determines which images should be removed from the analysis.

As the final step in the initial coregistration, the coefficients of the polynomial mapping function are inverted using the incidence matrix of the coregistration graph, such as in [2], [8]. Afterwards, all the images can be resampled onto the grid of the selected dataset reference image, given the polynomial mapping functions.

\section{GEOMETRICAL MAPPING FUNCTION CORRECTION USING PIXEL-OFFSET SERIES ON PT MATCH-PATCHES}

We recall that the purpose of the proposed coregistration strategy is to align all the images with the dataset reference image using geometrical mapping functions that are derived from satellite-orbit parameters and a DEM [13]. However, pixel-offsets derived from the satellite geometric information are usually far from amplitude-derived offsets because the satellite parameters are not entirely accurate. The low-frequency part of these differences can be explained by clock drifts of the SAR satellite [15], [17]. This error is usually modeled as a constant shift, and is corrected with pixel-offsets estimated from some tie coregistration patches [13], [14]. Nevertheless when we are processing multi-frame image pairs, the timing-parameter error can vary by a large amount along both the azimuth and range directions [15]. We therefore need to model the effects of clock drifts more accurately, by using more amplitude-derived offset estimations. One solution is that we can estimate pixel offsets by searching for the precise location of some isolated PTs, because the electromagnetic signatures of stable PTs are only slightly affected by the diversion of normal baselines [11], [19]. However, it can be difficult to find enough ideal PTs distributed throughout the whole scene when the image pair is acquired over a rural area. Rather than directly estimating pixel offsets between slave and reference images, we can detect match-patches with PTs and calculate the cross-correlation on them between SB image pairs, from which we can generate a pixel-offset series to correct the geometrical mapping functions.

The observed coherence depends mainly on geometrical and temporal components and we can therefore roughly obtain a temporal coherence map from the observed coherence values by dividing them by the geometrical coherence [20]. Targets with point-like features will exhibit high values (larger or close to one) in the decomposed temporal coherence map, as their real geometrical coherence values are larger than the values estimated under the distributed target assumption [19]. Then the PT match-patches can be detected by taking pixels with values close to one from 
the decomposed coherence map. This strategy works well when the temporal and normal baselines are small and we thus use the averaged decomposed coherence map associated with small baseline interferograms to detect PT match-patches. To ensure that good match-patches are distributed all over the image, the analysis is carried out on image blocks. Moreover if there are not enough high-coherence pixels in some blocks, we lower the threshold to retain a pre-assigned minimum number of pixels with highest coherence values. As the coherence calculation involves spatial averaging that reduces resolution, the detected match-patches may shift to surround the pixel with the highest amplitude (usually a PT). Therefore, we combine coherence and amplitude information to precisely locate PTs by finding the local amplitude maxima within the coherence estimation windows to position the matchpatches. Using the method described above, we detected 1369 match patches in the NAF dataset that are well distributed throughout the two-frame scene (Fig. 2a). The locations of the patches coincide with strongly reflecting pixels in the amplitude map. Not surprisingly, the area with the densest PT-patches is in and around Erzincan, a city located at the middle-left margin of the image (Fig. 2b). As the main goal here is to use pixel-offset series to correct the geometrical mapping functions but not to maximize the number of detected PTs in rural areas, one can employ any PT detection method without changing the proposed coregistration strategy.

After the PT match-patches have been detected, we can estimate the amplitude-derived pixel-offsets for each slave image with respect to the dataset reference image $\vec{\Delta}$ amp $\left(i^{r}, j^{r}\right)$. Given the coregistration connections, the estimation takes the following form: $A \vec{\Delta}_{\text {amp }}\left(i^{r}, j^{r}\right)=\vec{\Delta}_{\text {amp }}^{m, n}\left(i^{r}, j^{r}\right)$, i.e.:

$$
\left[\begin{array}{ccccc}
-1 & 1 & 0 & 0 & \ldots \\
-1 & 0 & 1 & 0 & \ldots \\
-1 & 0 & 0 & 1 & \ldots \\
0 & -1 & 1 & 0 & \ldots \\
\vdots & \vdots & \vdots & \vdots & \ddots
\end{array}\right]\left[\begin{array}{c}
\Delta_{\mathrm{amp}}^{1, r} \\
\Delta_{\mathrm{amp}}^{2, r} \\
\Delta_{\mathrm{amp}}^{3, r} \\
\vdots
\end{array}\right]=\left[\begin{array}{c}
\Delta_{\mathrm{amp}}^{1,2} \\
\Delta_{\mathrm{amp}}^{1,3} \\
\Delta_{\mathrm{amp}}^{1,4} \\
\Delta_{\mathrm{amp}}^{2,3} \\
\vdots
\end{array}\right]
$$

where for a PT match-patch located on the reference image $\left(i^{r}, j^{r}\right), \Delta_{\text {amp }}^{k, r}$ is the pixel-offset of $k$-th image with respect to the dataset reference and $\Delta_{\text {amp }}^{m, n}$ is the corresponding pixel-offset between $m$-th and $n$-th SAR images. Two conditions must be fulfilled to obtain the pixel-offset series for a match-patch: 1) all pixel-offsets $\vec{\Delta}_{\text {amp }}^{m, n}$ should be estimated on the same physical ground targets regardless of the image-pair $(m, n)$, and 2) the rank of the incidence matrix $A$ has to equal the number of images, i.e. all the images need to be connected in the coregistration. Then we can obtain the pixel-offset series with respect to the dataset reference image by inverting the incidence matrix $A$ for each PT:

$$
\overrightarrow{\boldsymbol{\Delta}}_{\mathrm{amp}}=\left(A^{T} A\right)^{-1} A^{T} \overrightarrow{\boldsymbol{\Delta}}_{\mathrm{amp}}^{m, n}\left(i^{r}, j^{r}\right)
$$

Examples of the obtained pixel-offset series for two PTs are shown in Fig. 3. In the range dimension, the main part of the residual pixel-offset is due to the smoothness of the polynomial mapping function, which does not consider topography. As a consequence, the pixel-offset series are correlated with the normal baseline [14] (Fig. 3b and d). In the azimuth dimension, large pixel-offset errors coincide with images with low quality indices (Figs. 1 
and 3a-c), implying that the overall interferogram coherence can be significantly reduced by a poor coregistration result.

As the PTs are detected from the coherence map derived from SB image pairs, we cannot guarantee that every match patch remains coherent during the whole time span of the dataset. It is then possible for some match patches that the coregistration graph is separated into several SB image sub-sets, i.e. the coregistration graph may not be fully connected. Therefore, before solving (4) for each PT, we have to update the incidence matrix $A$ by removing the pixel-offset measurements that cannot be referenced to the reference image, ensuring that $A$ is of full rank. The consequence is that for some PTs, only a part of the pixel-offset series can be estimated (Fig. 3c and d). Although removal of pixel-offset estimations from disconnected image pairs reduces the number of match-patches for some images, enough match patches should remain to update the geometrical mapping functions.

Besides the obtained pixel-offset series on the detected PTs, we then also calculate the pixel-offsets using the orbital information and a radar-coded DEM for each slave image [13]. The errors due to clock drifts on these PTs can be estimated from the difference between the amplitude and the geometrical-based pixel-offsets. We use a 2D polynomial model to fit the obtained pixel-offset differences, in particular when multi-frame images are processed. As an example, we took one image with 5-year separation from the dataset reference image and plot the clockrelated offsets as a function of azimuth and range coordinates (Fig. 4). The pixel-offsets in azimuth can vary about one pixel along both axes, and the range offsets also vary up to about half a pixel. This example demonstrates that a constant shift is insufficient in correcting the geometrical mapping function. Moreover, as shown by the black crosses in Fig. 4, directly estimating the pixel-offsets can be a challenge for rural test sites and therefore it is necessary to estimate the pixel-offset series through a SB configuration. As the final step, we calculate the azimuth and range pixel-offsets pixel-by-pixel using the corrected mapping function with which all the images are resampled onto the grid of the dataset reference image.

\section{VALIDATION}

The NAF is the main tectonic structure along the boundary between the Eurasian and Anatolian Plates. Several time-series InSAR analysis studies have been carried out over this region to measure the interseismic deformation across the fault [22]-[24]. However, rugged topographic and seasonal snow coverage significantly reduce the coregistration quality, and consequently lead to a low-density of coherent targets. To test our proposed coregistration strategy, we aligned a stack of 29 ASAR images (descending, track 35) using both a standard SB coregistration and our new method.

We note that the coherence is estimated using the spatial average operator, the improvement in coherence may therefore be underestimated. Since the stability of the amplitude is more sensitive to the quality of the coregistration, we evaluated our results by calculating the amplitude dispersion, $D_{A}$, which is used to indicate amplitude stability in many InSAR time-series analysis approaches [1], [2]. The $D_{A}$ values were calculated from the resampled images, which were coregistered using the two strategies. The results show a significant improvement in amplitude stability by using our new proposed method on images from this non-urban region. The number of pixels with $D_{A}$ values 
less than 0.25, a typical value for PS Candidates (PSC) [1], increased from 4977 to 11,231 . The minimum $D_{A}$ obtained also decreased from around 0.1 to 0.05 (Fig. 5a).

We also inspect the amplitude series of two typical and very stable scatterers $\left(D_{A}<0.15\right)$ as function of the normal baseline. In the first example (Fig 5b), the target amplitude is clearly related to the normal baseline after the standard SB coregistration (blue dots), while our method (red dots) shows stable amplitude. Because point-like targets show similar reflectivity coefficient when observed by SAR satellites from slightly different positions [19], this example suggests a point-like scatterer dominates the reflected radar signal within this resolution cell. It reveals that imprecise coregistration may not only lead to reduction in amplitude stability but also to misinterpretation of the scattering mechanism. The second example (Fig. 5c) shows similar amplitude stability improvement with a 33\% reduction of the amplitude dispersion $D_{A}$, from 0.18 to 0.12 .

\section{CONCLusions}

The new SAR image coregistration method clearly improves image coregistration in rural regions that suffer from seasonal feature changes and steep terrain. We achieve the improvement by introducing pixel-offset series to correct the geometrical mapping functions, and validate it with significant reduction in amplitude dispersion $\left(D_{A}\right)$. As a consequence, the number of permanent scatterer candidates doubled in the test case presented. This new coregistration strategy should therefore be helpful in improving time-series InSAR analysis in many applications. In addition, the proposed CQI provides accurate information about interferogram quality at the initial stage of time-series analysis. For sites where ground conditions are complex, e.g., seasonally covered by snow, the relative CQI helps in identifying a good dataset reference image and suitable interferometric combinations.

\section{REFERENCES}

[1] A. Ferretti, C. Prati, and F. Rocca, "Permanent scatterers in SAR interferometry," IEEE Trans. Geosci. Remote Sens., vol. 39, no. 1, pp. 8-20, Jan. 2001.

[2] A. Hooper, P. Segall, and H. A. Zebker, "Persistent scatterer interferometric synthetic aperture radar for crustal deformation analysis, with application to Volcán Alcedo, Galápagos," J. Geophys. Res., vol. 112, B07407, Jul. 2007.

[3] P. Berardino, G. Fornaro, R. Lanari, and E. Sansosti, "A new algorithm for surface deformation monitoring based on small baseline differential SAR interferograms," IEEE Trans. Geosci. Remote Sens., vol. 40, no. 11, pp. 2375-2383, Nov. 2002.

[4] T. Wright, B. Parsons, and E. Fielding, "Measurement of interseismic strain accumulation across the North Anatolian Fault by satellite radar interferometry," Geophys. Res. Lett., vol. 28, no. 10, pp. 2117-2120, May 2001.

[5] Q. Lin, J. F. Vesecky, and H. A. Zebker, "New approaches in interferometric SAR data processing," IEEE Trans. Geosci. Remote Sens., vol. 30, no. 3, pp. 560-567, May 1992.

[6] M. Liao, H. Lin and Z. Zhang, "Automatic registration of InSAR data based on least-square matching and multi-step strategy," Photogrammetric Eng. and Remote Sens., vol. 70, no. 10, pp. 1139-1144, Oct. 2004.

[7] R. F. Hanssen, Radar Interferometry. Data Interpretation and Error Analysis, Dordrecht, Kluwer Academic Publishers, 2001.

[8] A. Refice, F. Bovenga and R. Nutricato, "MST-based stepwise connection strategies for multipass radar data, with application to coregistration and equalization," IEEE Trans. Geosci. Remote Sens., vol. 44, no. 8, pp. 2029-2040, Aug. 2006.

[9] P. Marinkovic and R. F. Hanssen, "Advanced InSAR coregistration using point clusters," in Proc. IGARSS, 2004, vol. 1, pp. 489-492.

[10] R. Bamler and M. Eineder, "Accuracy of differential shift estimation by correlation and split-bandwidth interferometry for wideband and delta-k SAR systems,” IEEE Lett. Geosci. Remote Sens., vol. 2, no. 2, pp. 151-155, Apr. 2005. 
[11] F. Serafino, "SAR image coregistration based on isolated point scatterers," IEEE Lett. Geosci. Remote Sens., vol. 3, no. 3, pp. 354-358, Jul. 2006.

[12] D. Li and Y. Zhang, "A Fast Offset Estimation Approach for InSAR Image Subpixel Registration," IEEE Lett. Geosci. Remote Sens., vol. 9, no. 2, pp. 267-271, 2012.

[13] E. Sansosti, P. Berardino, M. Manunta, F. Serafino, and G. Fornaro, "Geometrical SAR image registration," IEEE Trans. Geosci. Remote Sens., vol. 44, no. 10, pp. 2861-2870, Oct. 2006.

[14] D. O. Nitti, R. F. Hanssen, A. Refice, F. Bovenga, and R. Nutricato, "Impact of DEM-assisted coregistration on high-resolution SAR interferometry," IEEE Trans. Geosci. Remote Sens., vol. 49, no. 3, pp. 1127-1143, Mar. 2011.

[15] T. Wang and S. Jónsson, "Phase-Ramp Reduction in Interseismic Interferograms from Pixel-Offsets," manuscipt submitted to IEEE Journal of Selected Topics in Applied Earth Observations and Remote Sensing.

[16] R. F. Hanssen and R. Bamler, "Evaluation of interpolation kernels for SAR interferometry," IEEE Trans. Geosci. Remote Sens., vol. 37, no. 1, pp. 318-321, Jan. 1999.

[17] D. Massonnet and H. Vadon, "ERS-1 internal clock drift measured by interferometry," IEEE Trans. Geosci. Remote Sens., vol. 33, no. 2, pp. 401-408, Mar. 1995.

[18] P. Misra, B. P. Burke, and M. M. Pratt, "GPS performance in navigation,” Proceedings of the IEEE, vol. 87, no. 1, pp. 65-85, Jan. 1999.

[19] D. Perissin, C. Prati, M. E. Engdahl, and Y. L. Desnos, "Validating the SAR wavenumber shift principle with the ERS Envisat PS coherent combination," IEEE Trans. Geosci. Remote Sens., vol. 44, no. 9, pp. 2343-2351, Sep. 2006.

[20] T. Wang, M. Liao, and D. Perissin, "InSAR coherence-decomposition analysis," IEEE Lett. Geosci. Remote Sens., vol. 7, no. 1, pp. 156-160, Jan. 2010.

[21] F. Casu, A. Manconi, A. Pepe, and R. Lanari, "Deformation time-series generation in areas characterized by large displacement dynamics: the SAR amplitude pixel-offset SBAS technique," IEEE Trans. Geosci. Remote Sens., vol. 49, no. 7, pp. $2752-2763$, Jul. 2011.

[22] R. J. Walters, R. J. Holley, B. Parsons, and T. J. Wright, "Interseismic strain accumulation across the North Anatolian Fault from Envisat InSAR measurements," Geophys. Res. Lett., vol. 38, no. 5, pp. 1-5, Mar. 2011.

[23] M. Motagh, J. Hoffmann, B. Kampes, M. Baes, and J. Zschau, "Strain accumulation across the Gazikoy-Saros segment of the North Anatolian Fault inferred from Persistent Scatterer Interferometry and GPS measurements," Earth and Planet. Sci. Lett., vol. 255, no. 3-4, pp. 432-444, Mar. 2007.

[24] O. Cavalié and S. Jónsson, "Block-like plate motions in eastern Anatolia," manuscipt submitted. 


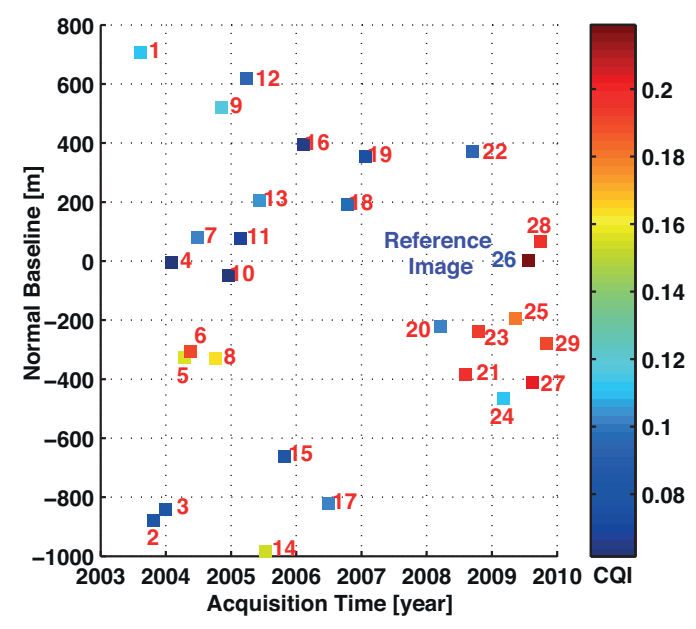

Fig. 1. Coregistration Quality Index (CQI) for each SAR image derived from the initial coregistration. Each colored square represents an image in the spatial-temporal baseline plane of the test dataset. Image 26 was selected as the dataset reference image because it has the highest quality index. 


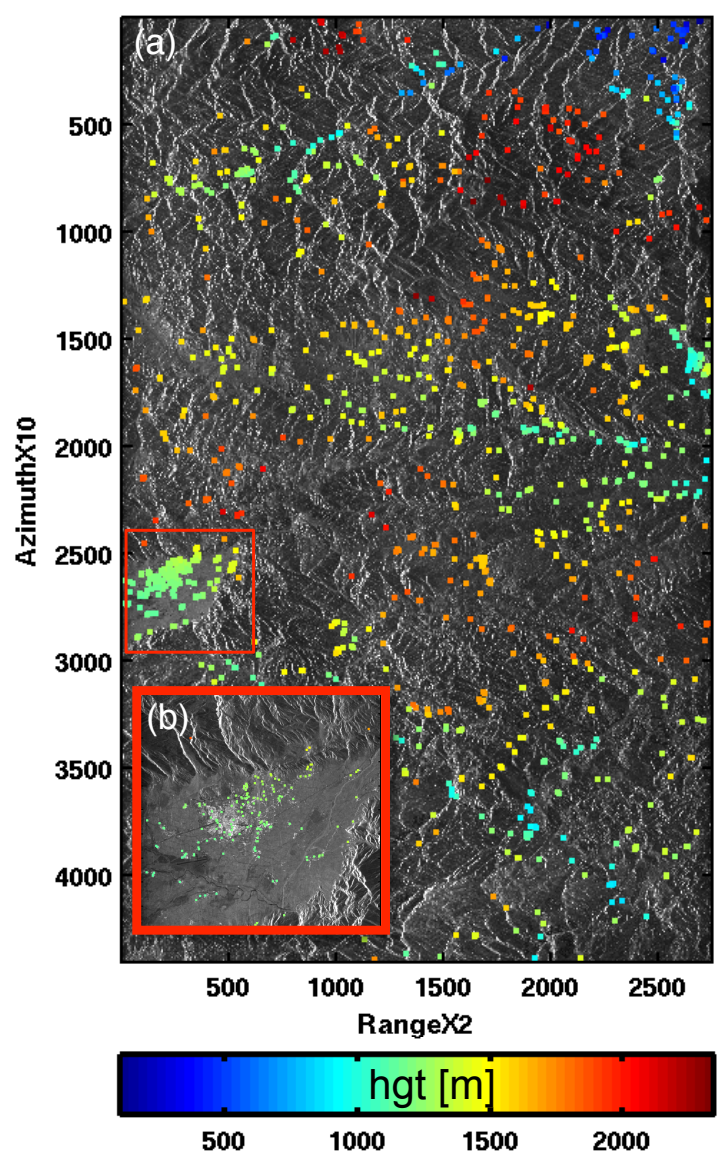

Fig. 2. (a) Point-like match-patches detected for the North Anatolian Fault test site. They are plotted on the incoherent mean amplitude map (10 by 2 multilooked) with the patch color indicating elevation. (b) is a zoom-in view of the red rectangle shown in (a) covering the city of Erzincan.

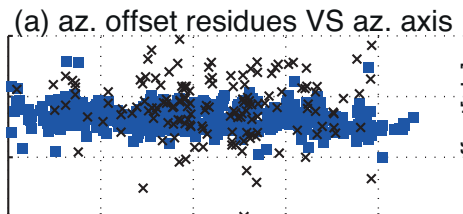

(c) ra. offset residues VS az. axis



(b) az. offset residues VS ra. axis



(d) ra. offset residues VS ra. axis

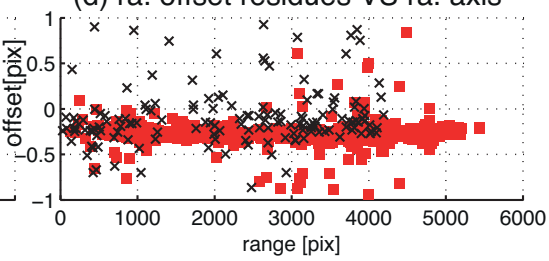

Fig. 3. Pixel-offset series for two detected PTs in azimuth (blue dots) and in range (red dots) as a function of normal baseline. The black dots in (c) and (d) indicate images isolated by the coregistration graph for PT \#1123. 


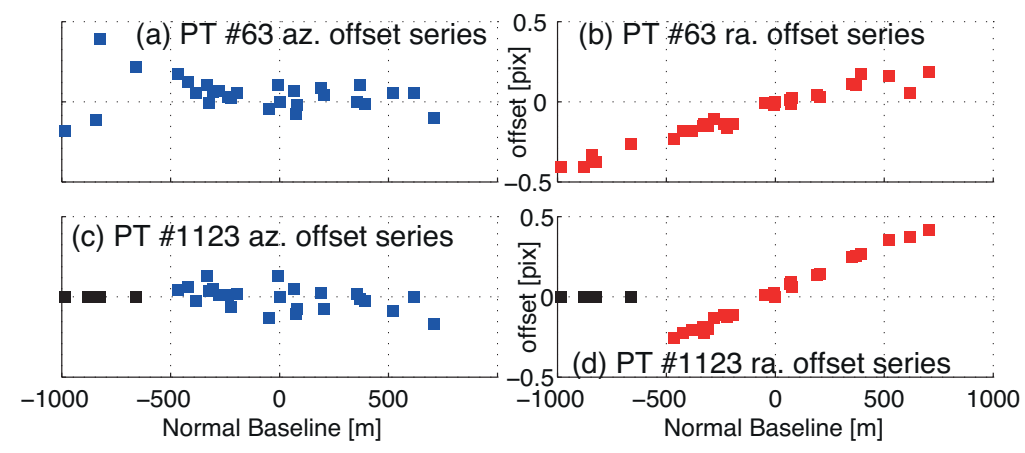

Fig. 4. Geometrical mapping function residues (in pixels) as a function of the azimuth and range directions, derived from the difference between geometric offsets and amplitude-based pixel-offset series. They are obtained for image \#4 w.r.t. the dataset reference image (Fig. 1). (a) and (b) show changes of the azimuth pixel-offset residues along the azimuth and range directions, while (c) and (d) show the range residues along the two directions. The black x-marks indicate the pixel-offsets between these two images directly estimated on the detected point-like targets [11].
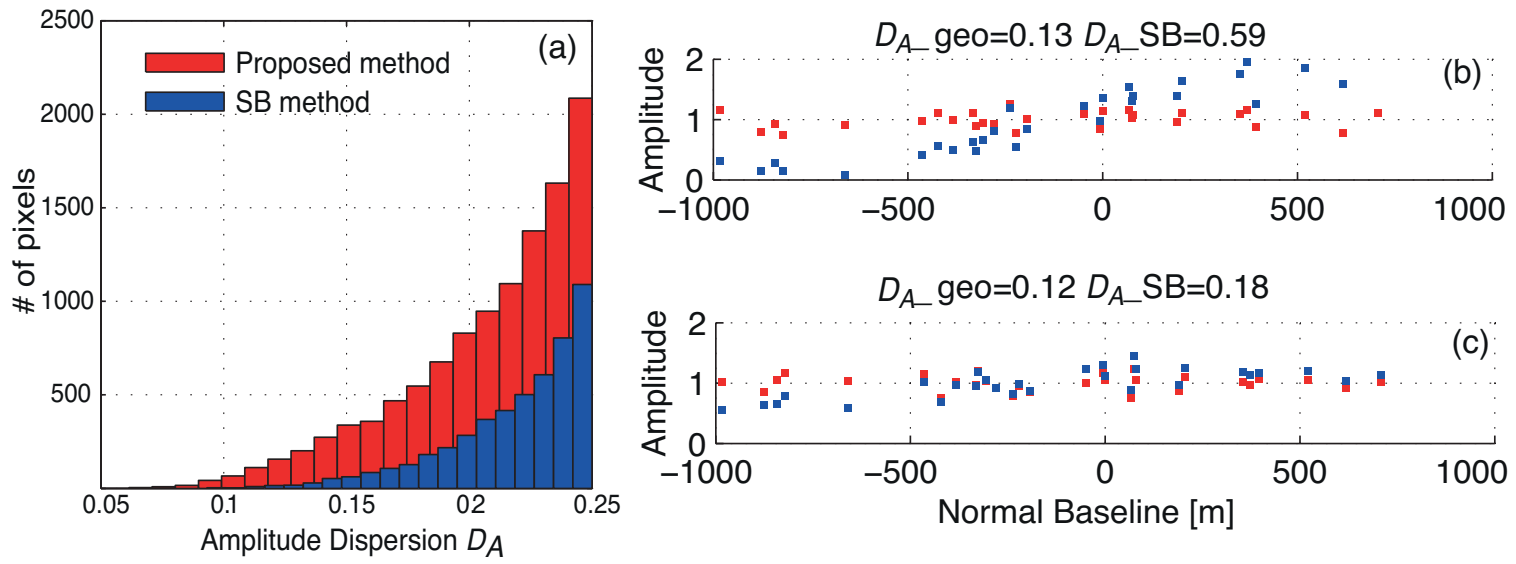

Fig. 5. (a) Histograms of pixels with amplitude dispersion $\left(D_{A}\right)$ values smaller than 0.25 for the North Anatolian Fault images after a standard SB-coregistration (blue) and the presented method (red). (b) and (c) show amplitude series w.r.t. normal baseline for two very stable pixels $\left(D_{A}<0.15\right)$ after the two coregistration strategies with $D_{A \_} \mathbf{S B}$ and $D_{A \_}$geo as their corresponding $D_{A}$ values. 